\title{
Right Ventricular Bifocal Stimulation in the Treatment of Dilated Cardiomyopathy with Heart Failure
}

\author{
José Carlos Pachón Mateos, Remy N elson Albornoz, Enrique Indalécio Pachón Mateos, Vera Márcia \\ Gimenez, Juán Carlos Pachón Mateos, Maria Zélia Cunha Pachón, Eusebio Ramos dos Santos Fo, \\ Paulo de Tarso Jorge Medeiros, Marco Aurélio Dias da Silva, Paulo Paredes Paulista, José Eduardo \\ Moraes Rêgo Sousa, Adib Domingos Jatene
}

São Paulo, SP - Brazil

Objective - To describe a new more efficient method of endocardial cardiac stimulation, which produces a narrower $Q R S$ without using the coronary sinus or cardiac veins.

Methods - We studied 5 patients with severe dilated cardiomyopathy, chronic atrial fibrillation and AV block, who underwent definitive endocardial pacemaker implantation, with 2 leads, in the RV, one in the apex and the other in the interventricular septum (sub pulmonary), connected, respectively, to ventricular and atrial bicameral pacemaker outputs. Using Doppler echocardiography, we compared, in the same patient, conventional (VVI), high septal ("AAI") and bifocal ("DDT" with AV interval $\cong 0$ ) stimulation.

Results - The RV bifocal stimulation had the best results with an increase in ejection fraction and cardiac output and reduction in $Q R S$ duration, mitral regurgitation and in the left atrium area $(p \leq 0.01)$. The conventional method of stimulation showed the worst result.

Conclusion - These results suggest that, when left ventricular stimulation is not possible, right ventricular bifocal stimulation should be used in patients with severe cardiomyopathy where a pacemaker is indicated.

Keywords: pacemaker, multisite stimulation and heart failure

Instituto Dante Pazzanese de Cardiologia and Hospital do Coração - São Paulo Maling address: José Carlos Pachón Mateos - Av. Jamaris, 650/72 - 04078-001 São Paulo, SP - Brazil
In dilated cardiomyopathy some degree of delay occurs in myocardial stimulus conduction, which causes QRS widening. Associated lesions in the conduction system also often cause QRS widening. In these cases, when a cardiac pacemaker is necessary, paced QRS is more enlarged, easily achieving $200 \mathrm{~ms}$ or even more. The delayed ventricular activation, by itself, provokes systolic and diastolic dysfunction, and increases mitral regurgitation ${ }^{1}$.

Since the beginning of cardiac pacing, it has been known that the contraction caused by a paced QRS is less effective than the one resulting from a normal QRS. When the QRS is wide, the increased pressure caused by the first stimulated myocardium area is lessened by the natural complacence of other areas that will be activated later. On the other hand, in the normal contraction, the fast myocardial cell activation creates a mechanical synergism, extremely favorable for taking maximum advantage of the inotropic state. It causes a pressure wave with high $\mathrm{dP} / \mathrm{dt}$, which is a faster, highly efficient rise in pressure. In the dilated myocardium, the activation generated by a pacemaker is distributed over a longer time, causing a pressure wave that is more attenuated proportionally to the paced QRS widening. To preserve systolic and diastolic functions, and reduce mitral insufficiency, it appears to be fundamental to pace both ventricles with a normal QRS, or at least with the shortest PRS possible. This can be easily obtained by AAI pacing, when the patient has intra- and atrioventricular conduction systems preserved. In the case of AV block, the resulting ventricular paced QRS (almost always placed on the right ventricle) is very wide. It is possible to have a narrow QRS simultaneously pacing more than one point. Recent studies have shown narrow QRS and improved myocardial contractility, when both ventricles are simultaneously paced ${ }^{2}$. The problem is access to the left ventricle. The first approach was epicardial, which requires a thoracotomy ${ }^{3}$. The alternative is the use of cardiac veins; through the coronary sinus. This method avoids thoracoto- 
my, because of the endocardial access. But technical difficulties related to the great anatomical variety of cardiac veins sometimes impede the implantation.

The main purposes of this work were: 1) To suggest an easy way for ventricular pacing, with narrower QRS, through the placement of two leads in the right ventricle, using an endocardial access, without a thoracotomy or coronary sinus access; 2 ) to propose a study method that allows the testing of hemodynamic efficiency of this kind of pacing compared with conventional heart stimulation, in the same patient, eliminating intervening variables that could occur among different individuals, and permit answers to the following questions: a) Is it possible to partially "resynchronize" the left ventricular myocardium, stimulating only the right ventricle? b) The resulting "resynchronization degree" has some beneficial effects in the heart stimulation in cases of severe heart failure of dilated cardiomyopathy? c) Is the implantation technique safe, reproducible and easy to perform with low risk in critical patients?

\section{Methods}

Five patients ( 1 female) with cardiac pacemaker indication were included. Ages ranged from 37 to 66 years (mean of 52.2 \pm 17.7 years). All patients had severe dilated cardiomyopathy (class III and IV NYHA), caused by Chagas' disease in 4 cases and idiopathic in one case. All patients had atrial fibrillation with high degree or complete AV block and cardiac failure with radiological cardiac shapes 3 to 4+.

Implantation techniques - Two endocardial leads were implanted in the right ventricle. The first lead ("septal") was implanted in the His bundle area, and the second lead was placed in the right ventricle apex according to the classical endocardial implantation technique (figure 1). The pacemaker used in this series was the Biotronik Dromos (DDDR pacemaker that allows AV delay of $15 \mathrm{~ms}$, in the DDT mode). Ideally, the pacemaker should be able to use $0 \mathrm{~ms}$ of AV delay. The "septal" lead was connected to the atrial channel of the pacemaker and the conventional lead to the ventricular channel. To avoid the risk of pacemaker syndrome (due to ventricular stimulation) only patients with chronic atrial fibrillation and AV block were selected.

Programming and echocardiographic study - All patients had cardiac insufficiency. Therefore, after the implant the pacemakers were programmed in "DDT" mode, with an AV delay of $15 \mathrm{~ms}$ (bifocal VVI pacing). After 2 weeks of evolution, with heart rate corrected by artificial pacing, all patients were evaluated by echocardiography in three pacing modes: 1) By programming the pacemaker to AAI mode (septal stimulation), the His bundle area, or the right ventricle outflow area- was stimulated;2) By programming the pacemaker to VVI mode, conventional pacing was obtained and; 3) By programming the pacemaker to DDT mode (AV delay $=15 \mathrm{~ms}$ ), it was possible to stimulate two points (high Septum and Apex), of right ventricle, almost simultaneously (figure 2). Our objective was to stimulate at the same time two distant points of the right ventricle.

All programming was performed in the echocardiography laboratory by maintaining the same heart rate. The patient was under absolute rest, without positional changes. After allowing 5 minutes for hemodynamic stabilization ehocardiographic parameters were measured. Subsequently, mono and bidimensional Doppler modes were used to measure ejection fraction (EF), cardiac output (CO), functional mitral regurgitation area (MR) and length of QRS complex (in 3 simultaneous derivations DI, DII and DIII). Average values and standard deviation were calculated, using paired $t$ test for equivalent variance series, comparing septal and bifocal right ventricular stimulation with conventional stimulation.

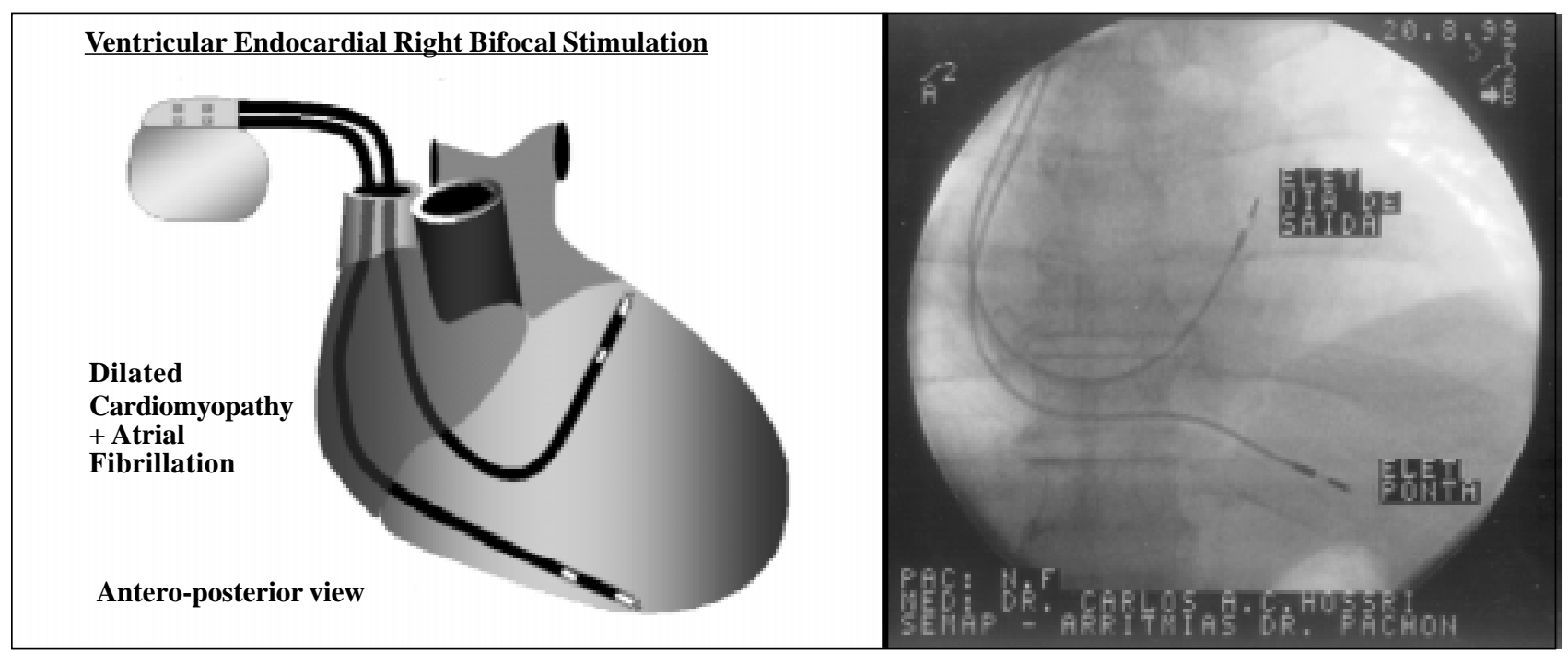

Fig. 1 - Left panel: scheme of the leads' positions for the 3 stimulation modes used in this study: right ventricular septal, bifocal and apex (conventional). Right panel: radioscopy showing the positions of the leads in a definitive bifocal system. 


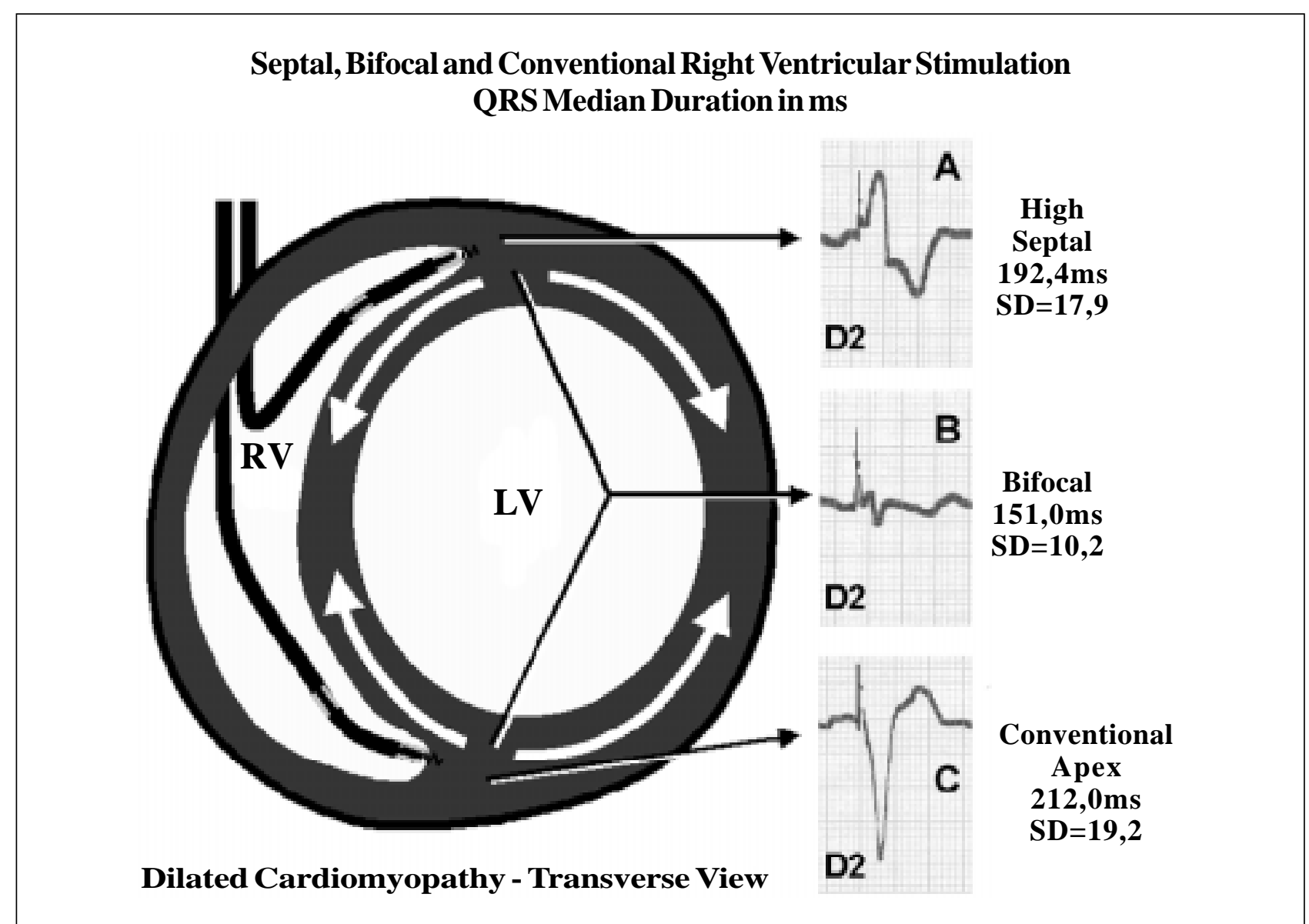

Fig. 2 - Scheme of the 3 stimulation modes used and tested in this study model with the stimulated QRS, the mean of durations and the standard deviation in the studied group A, B and C. The stimulated QRS resulting from the bifocal stimulation is clearly narrower (B).

\section{Results}

Narrowing the QRS - With bifocal stimulation, the mean duration of the QRS was $151 \mathrm{~ms}(\mathrm{SD}=10.2)$, in other words, 61 $\mathrm{ms}$ narrower in relation to the mean QRS duration of the conventional stimulation $(212 \mathrm{~ms}, \mathrm{SD}=19.2)$. This narrowing was statistically significant $p=0.003$. The septal pacing also showed a narrower QRS than conventional pacing, however without statistical significance (table I and figure 2).

Echocardiographic parameters - Comparing the conventional stimulation with the bifocal stimulation, it was verified that all the echocardiographic parameters had significant improvement. The average data show that the ejection fraction increased $6.8 \%(p=0.002)$, and the cardiac output increased $0.61 / \min (p=0.01)$. Similarly, there was a significant reduction of the left atrium, average $7.5 \mathrm{~cm}^{2}$ $(p=0.004)$ due to an evident reduction in the degree of functional mitral regurgitation. The regurgitation area was reduced to an average of $7.4 \mathrm{~cm}^{2}(p=0.006)$.

\section{Discussion}

Because short AV delay was not clearly useful in the treatment of the heart failure, a greater interest exists in "ventricular resynchronization".
Mechanically, wide QRS (common in the severe cardiomyopathy and in ventricular pacing by artificial pacemaker) (figure 2), is clearly less effective than narrow QRS ${ }^{5,6}$. The more enlarged the QRS, the greater the harm to contractility. The activation of all myocardial cells almost at the same time causes a synergic action with a contraction of great mechanical efficiency. However, in the presence of slow conduction, other areas that will only be activated later lessen the contraction of the myocardial region. When the heart is very dilated, this phenomenon is more important, contributing to contractile dysfunction, in addition to the cardiomyopathy by itself (figure 3). Besides the systolic dysfunction, the enlargement of QRS provokes an increase in functional mitral regurgitation and diastolic dysfunction ${ }^{7}$, reducing ventricular filling time.

Several studies ${ }^{8}$ about process, test the viability and clinical usefulness of "ventricular resynchronization", stimulating the right and left ventricle at the same time. Left ventricular stimulation, however, faces some technical difficulties. The endocardial approach has to be performed in the coronary sinus, through cardiac veins. Otherwise, it must be performed by epicardial access with a thoracotomy. Chronic endocardial left ventricular stimulation through a transseptal puncture is not advisable due to the risk of 


\begin{tabular}{|ccccccccccccccccc|}
\hline \multicolumn{110}{c|}{ Table I - Echocardiographic parameters } \\
\hline Pt & EF/S & EF/C & EF/BF & CO/S & CO/C & CO/BF & LAS & LA/C & LA/BF & MR/S & MR/C & MR/BF & QRS/S & QRS/C & QRS/BF \\
\hline 1 & 0.09 & 0.13 & 0.18 & 2.26 & 2.35 & 2.42 & 32.3 & 49.4 & 36.4 & 24.1 & 31 & 20.2 & 185 & 220 & 140 \\
2 & 0.27 & 0.32 & 0.35 & 3.7 & 3.58 & 3.99 & 29.2 & 34 & 27.1 & 12.1 & 14 & 7.1 & 200 & 190 & 155 \\
3 & 0.17 & 0.18 & 0.27 & 2.39 & 1.99 & 2.97 & 18.1 & 22.1 & 18.3 & 8.5 & 7.5 & 6.3 & 175 & 240 & 160 \\
4 & 0.34 & 0.27 & 0.35 & 3.65 & 3.19 & 3.93 & 7.11 & 12.8 & 3.93 & 5.6 & 10.7 & 3.48 & 220 & 200 & 160 \\
5 & 0.32 & 0.28 & 0.37 & 3.2 & 2.9 & 3.9 & 18.2 & 24.1 & 19.1 & 7.6 & 12.5 & 5.5 & 182 & 210 & 140 \\
M & 0.24 & 0.24 & 0.30 & 3.0 & 2.8 & 3.4 & 21.0 & 28.5 & 21.0 & 11.6 & 15.1 & 8.5 & 192.4 & 212.0 & 151.0 \\
DP & 0.11 & 0.08 & 0.08 & 0.7 & 0.6 & 0.7 & 10.1 & 13.9 & 12.0 & 7.4 & 9.2 & 6.7 & 17.9 & 19.2 & 10.2 \\
P & 0.468 & & 0.002 & 0.038 & & 0.011 & 0.018 & & 0.005 & 0.032 & & 0.006 & 0.275 & & 0.003 \\
\hline
\end{tabular}

EF- ejection fraction; S- RV septal stimulation; C- RV conventional stimulation (right ventricular apex); CO- cardiac output (1/min), BF: RV bifocal stimulation between the high septal region and right ventricular apex; LAA- left atria area $\left(\mathrm{cm}^{2}\right)$; MR- functional mitral regurgitation area $\left(\mathrm{cm}^{2}\right)$; QRS- length of the QRS measured in 3 simultaneous ECG leads (I, II, III); SD- standard deviation; p- p-value (paired t test comparing septal and bifocal right ventricular stimulation with the conventional stimulation).

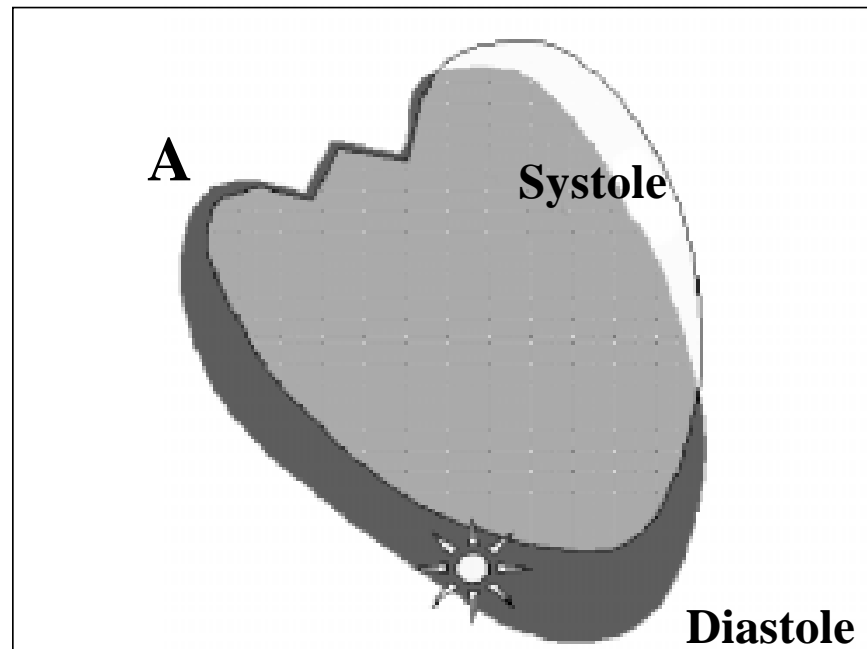

\section{Conventional Stimulation$$
\text { QRS = } 200 \mathrm{~ms}
$$$$
\text { Inotropic asynergism }
$$

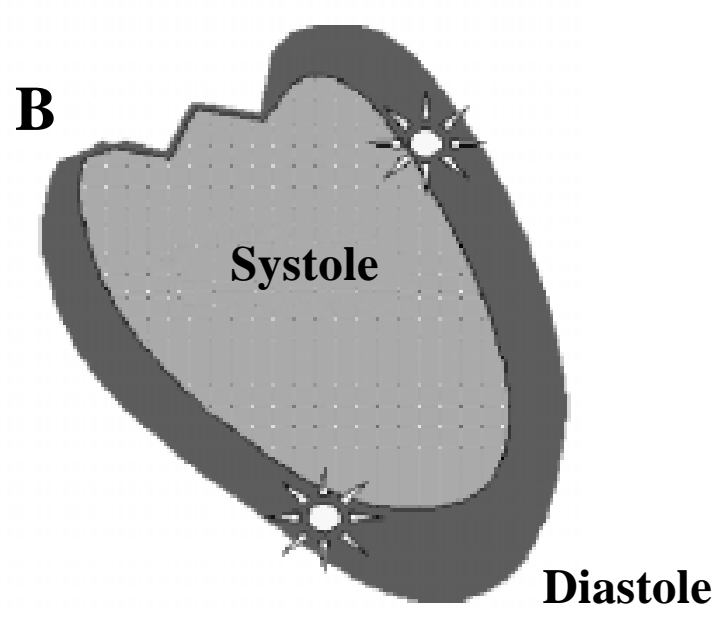

Bifocal Stimulation

QRS = $150 \mathrm{~ms}$ Inotropic synergism

Fig. 3 - Scheme of the contraction damage caused by the lack of inotropic synchronism due to very slow myocardial conduction. When the delayed region was activated, myofibril interposition was reduced by the contraction of the initial activated areas. The loss of optimal myofibril interposition and the activation when the intraventricular pressure is increasing are strong factors that appose the contractility.

systemic thromboembolism. Stimulation through cardiac veins, in addition to the access difficulty, causes these additional problems: the need of a special lead for cardiac veins, long-term stability of the lead, the tendency to higher stimulation thresholds, cardiac vein phlebitis, problems with removing chronicle leads, impossibility of access in cases where anatomical variations are present. On the other hand, left ventricular epicardial stimulation classically has higher acute and chronic thresholds, plus imposing the need of a thoracotomy, which is highly undesirable in patients with congestive heart failure, with severe and definitive cardiomyopathy, who are therefore at high surgical risk.

Due to these considerations, we decided to do a study proposing a simple form of definitive right endocardial ventricular stimulation with narrower QRS in dilated cardiomyopathy and a protocol for answering the following questions:

Ventricular resynchronization - The results suggest that it is possible to partially resynchronize the ventricular myocardium with bifocal right ventricular pacing, resulting in a significant narrowing of QRS, and the improvement of cardiac contractility (figure 4 ).

Hemodynamics improvements- The comparison of three stimulation modes showed that the best hemodynamic efficiency was obtained with right bifocal stimulation. Comparison of conventional with bifocal stimulation reveals a significant increase in ejection fraction $(+28.8 \%, p=0.0024)$, 


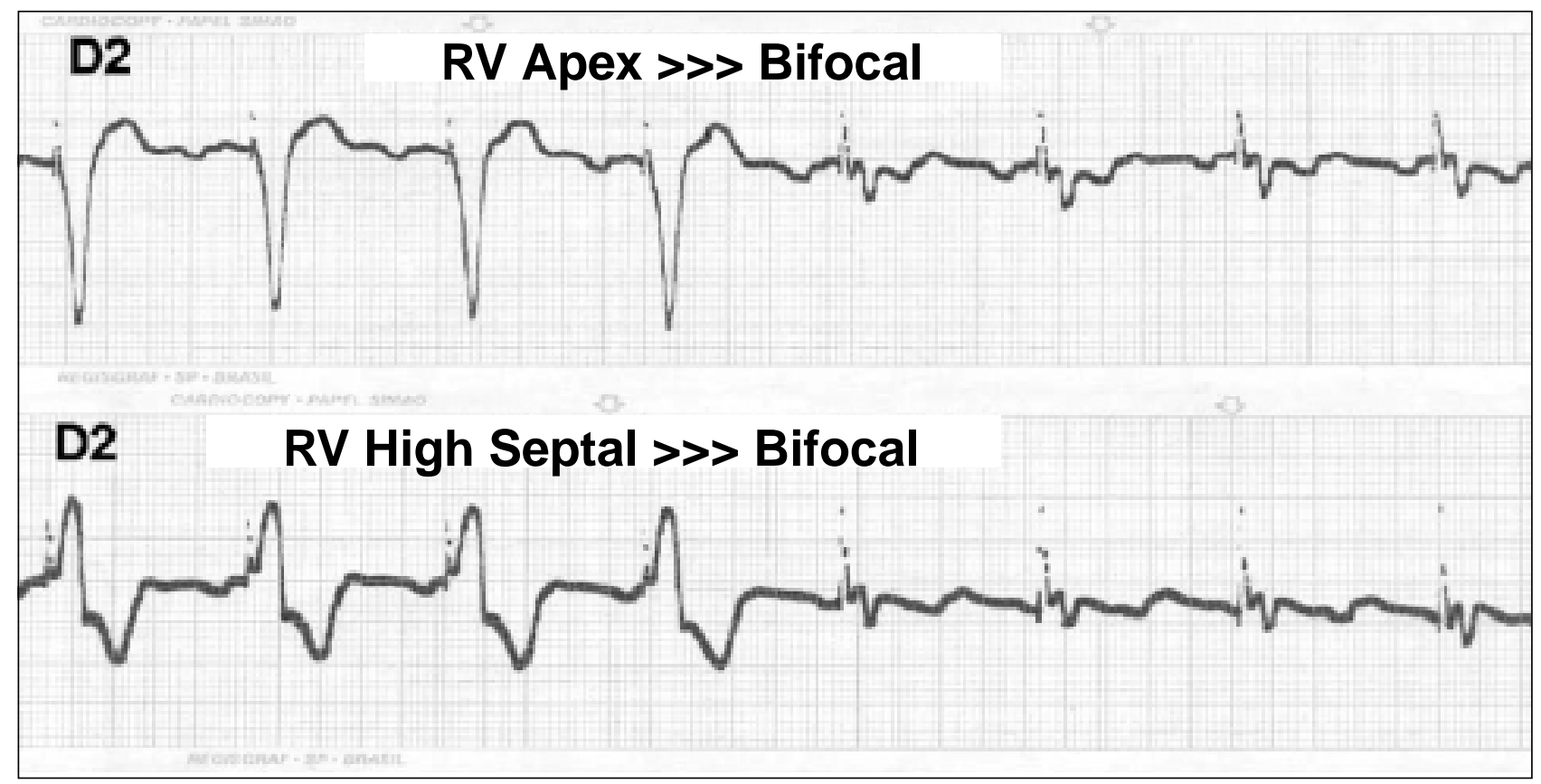

Fig. 4 - Impact of the right ventricular bifocal stimulation in the QRS narrowing. In this example the QRS from $240 \mathrm{~ms}$ in the conventional pacing narrowed to $150 \mathrm{~ms}$ in the bifocal stimulation. After 8 days the hemodynamic atrial improvement provided conditions for chronic atrial fibrillation reversion to atrial tachycardia.

cardiac output $(+21.4 \%, p=0.0114)$, a significant narrowing of QRS(-28.8\%, $p=0.003)$, mitral regurgitation $(-43.7 \%, p=0.0063)$ and left atrium area $(-26.3 \%, p=0.0049)$ (table I). A very interesting outcome was the significant reduction in mitral regurgitation. We believe that this highly desirable effect is due to the partial resynchronization of ventricular contraction, which favors mitral function (fig. 5). Except by the ejection fraction ( $p=0.468)$, the high septal stimulation also showed a better efficiency than conventional stimulation, but hemodynamic benefit was less evident when compared with that of bifocal stimulation (table I).

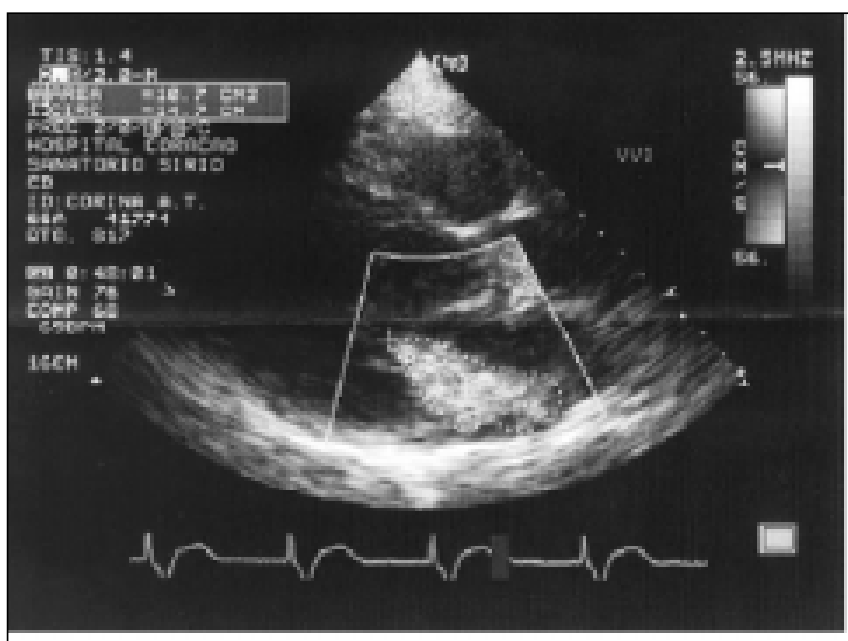

\section{Conventional stimulation}

Implantation technique - The implantations were performed following the classical methodology of the endocardial bicameral pacemakers with both leads by the venous route, and easily reproducible. The first lead was positioned, preferably in the apex of the right ventricle (the most distant possible of the base). The second lead was positioned in the His bundle area, guided by the H potential and by anatomical elements. In four patients, due to severe cardiomyopathy, the $\mathrm{H}$ potential was difficult to locate and, in that case, the lead was positioned in the subpulmonary region (figure 6). This position was easily obtained by

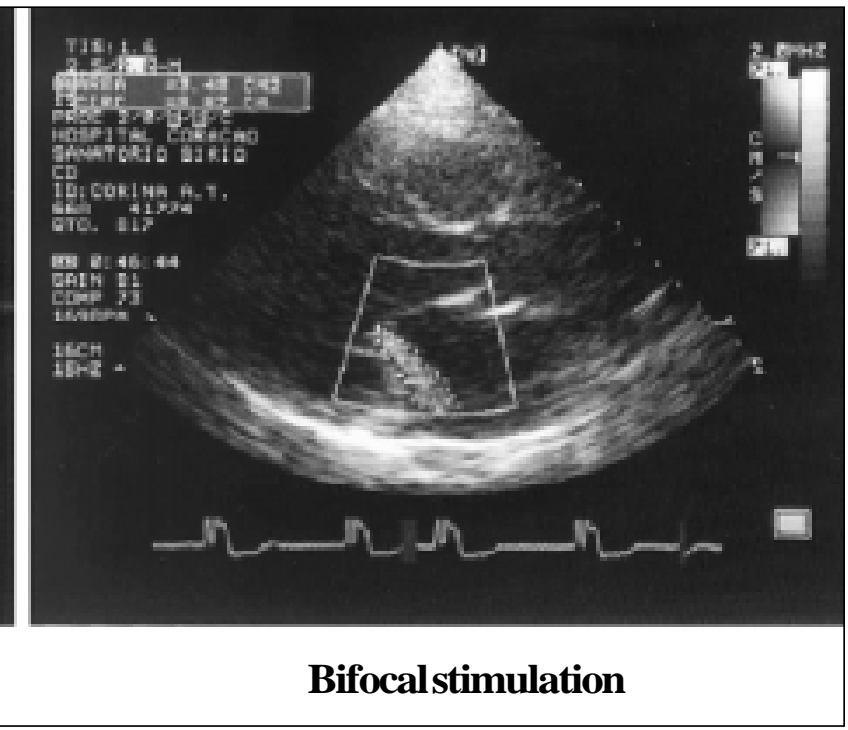

Fig. 5 - Bidimensional echocardiogram showing significant reduction in functional mitral regurgitation with right ventricular bifocal stimulation. In addition to the reduction in regurgitation, evident change is also verified in the direction of the regurgitating flow, suggesting that the change in the ventricular activation modifies and favors synchronism among the mitral papillary muscles. 


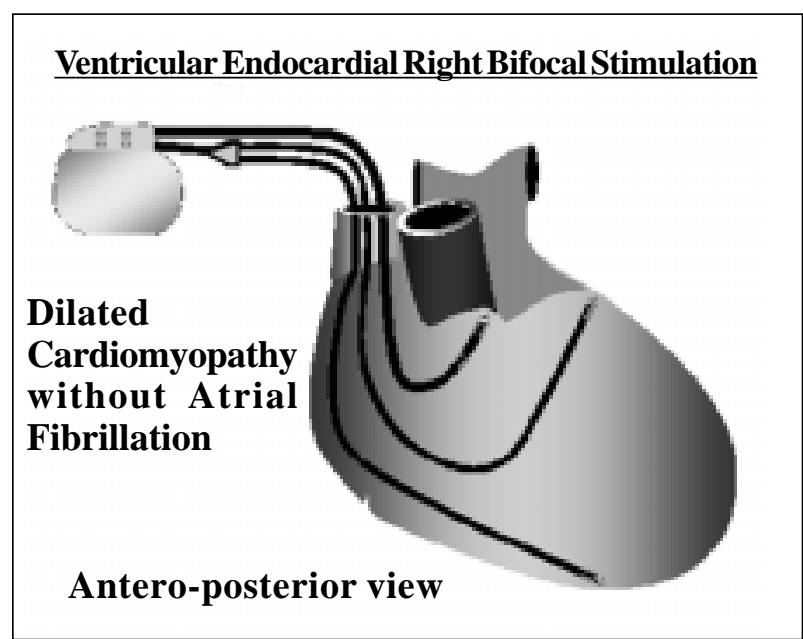

Fig. 6 - Stimulation suggested by the authors to take advantage of the benefit of the bifocal right ventricular endocardial pacing in a patient without atrial fibrillation. In the ventricles, new generation thin unipolar leads may be used.

entering in the pulmonary artery, slowly withdrawing the lead that was previously connected to the stimulator until ventricular capture was obtained. The left anterior oblique position was used to advance the tip of the lead at its maximum, through the left ventricle. In all cases, this lead was bipolar, active fixation. The $\mathrm{R}$ wave, impedance and thresholds were obtained using conventional techniques. The first lead (RV apex) was connected to the ventricular channel, and the second lead (septal - His bundle - RV outflow), was connected to the atrial channel of the pulse generator. Lead fixation and pocket closure were performed using conventional techniques. Finally, keeping in mind that AV synchronicity significantly contributes in the efficiency of an insufficient heart ${ }^{9}$, we used the pacing configuration shown in figure 6 , in the dilated cardiomyopathy, functional mitral insufficiency and AV block, without atrial fibrillation.

Clinical evaluation - In the short-term (mean follow-up of 5.6 \pm 1.1 months) a significant clinical improvement occurred in all cases that changed from NYHA class III and IV to class II (fig. 7). A long-term clinical follow-up will be very important to evaluate the constancy of the results.

Critical considerations - To obtain definitive information, the number of patients in this study is obviously low. However, each patient was his or her own control, which is a particular type of study that allows obtaining results with a smaller number of patients. In addition, our initial objective was mainly to find trends and study models that can be useful in extensive new projects.

We believe that the positive point of this study is the proposed model. This model used ventricular stimulation (VVI) without harm because all patients had chronic atrial fibrillation. This way, without any technical barrier, we can use the bicameral pacemaker DDD to obtain unquestionable and safe long-term information, of the multisite ventricular stimulation. Even before the definitive results, a clear benefit to the patients is revealed in the measure that they receive safer stimulation (two independent stimulation points), which is certainly physiologically preferable (narrower QRS). In fact, based on

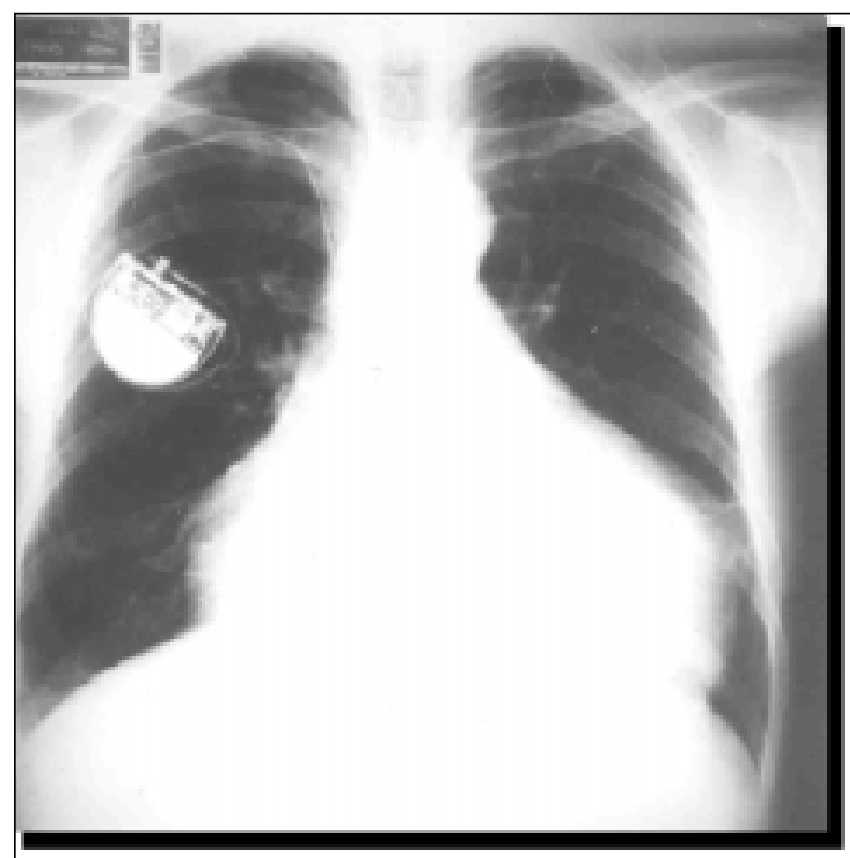

Conventionalstimulation

(9 years)

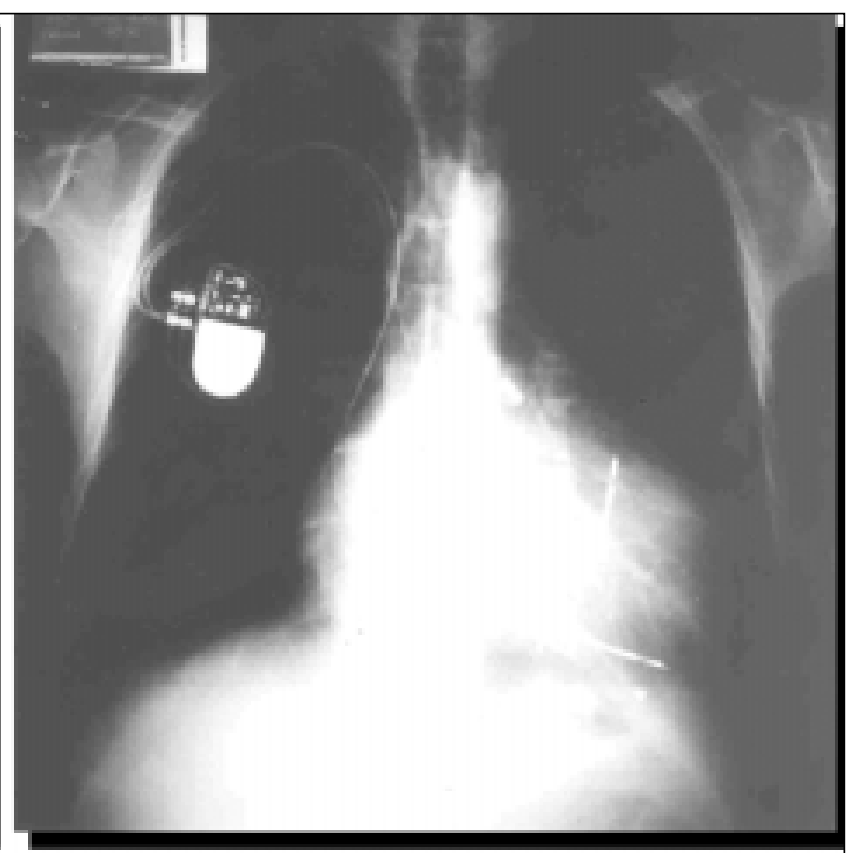

Bifocal stimulation

(8 days)

Fig. 7 - Thoracic X-rays obtained in the same patient, the first with nine years of evolution with conventional ventricular pacing and the second, after 8 days of bifocal ventricular stimulation. There was an evident reduction in heart size. In this case the old VVI pacemaker was changed due to battery depletion, a bifocal system being implanted by adding a septal lead. 
these arguments we usually adopt this stimulation method for the cases of AV nodal ablation for refractory atrial tachyarrhythmia treatment. In case of exit block in the conventional lead, the other lead maintain ventricular stimulation.

The comparison of echocardiographic variables, changing the ventricular stimulation points through noninvasive programming, without any change in patient position, heart frequency, respiratory frequency, physical activity, venous return, sympathetic tone or psychological condition, comprises a valuable method capable of achieving minimum hemodynamic modifications.

Another critical feature is the ability to control by echocardiogram alone. This is justified by the fact that our initial objective was to find clear and immediate evidence that justified the accomplishment of a larger number of implants without any harm to patients.

Conclusion - In this group of patients with severe dilated cardiomyopathy and functional mitral insufficiency, under echocardiographic evaluation of the stimulation modes tested, the classic conventional mode was the worst. The bifocal stimulation, easily obtained in the right ventricle, was significantly superior in all tested parameters. There was a good and evident "intra-ventricular resynchronization" with better contractility caused by a significant reduction in QRS duration. The implantation technique was simple and did not cause an increase in surgical risk.

\section{References}

1. Xiao HB, Brecker SJ, Gibson DG. Effects of abnormal activation on the time course of the left ventricular pressure pulse in dilated cardiomyopathy. Br Heart J 1992; 68: 403-7.

2. Gras D, Mabo P, Tang T, et al. Multisite pacing as a supplement treatment of congestive heart failure preliminary results of the Medtronic Inc. InsSync Study. PACE 1998; 21: 2249-55.

3. Cazeau S, Ritter P, Bakkdach S, et al. Four chamber pacing cardiomyopathy. PACE 1993; 17: 1974-9.

4. Buckingham TA, Candidas R, Fromer M, et al. Acute hemodynamic effects of atrioventricular pacing at differing sites in the reight ventricle individually and simultaneously. PACE 1995; 18: 1772.

5. Prinzen FW, Augusstijn CH, Allessie MA, et al. The time sequence of electrical and mechanical activation during spontaneous beating and ectopic stimulation. Eur Heart J 1992; 13: 535-43.

6. Bakker PF, Meijburg H, de Jonge N, et al. Benefiicial effects of biventricular pacing in congestive heart failure. PACE 1994; 17: 820.

7. Xiao HB, Lee CH, Gibson DG. Effect of left bundle branch block on diastolic function in dilated cardiomyopathy. Br Heart J 1991; 66: 443-7.

8. Foster AH, Gold MR, Mc Laughlin JS. Acute hemodynamic effects of atriobiventricular pacing in humans. Ann Thorac Surg 1995; 59: 294-300.

9. Nishimura RA, Hayes DL, Holmes DR, et al. Mechanism of hemodynamic improvement by dual-chamber pacing for severe left ventricular dysfunction: An acute Doppler and catheterization hemodynamic study. J Am Coll Cardiol $1995 ; 25: 281-8$. 